\title{
Burden of ESBL with antibiogram in clinical isolates of E.coli and Klebsiella species from tertiary care hospital in central India
}

\author{
Surender Kaur ${ }^{1, *}$, Abhijit Awari ${ }^{2}$ \\ ${ }^{\mathbf{1}}$ Assistant Professor, ${ }^{2}$ Professor and HOD, Dept. of Microbiology, Raipur Institute of Medical Sciences, Raipur, Chhattisgarh, \\ ${ }^{2}$ Padmashri Dr Vithalrao Vikhe Patil Foundation Medical College, Ahmednagar, Maharashtra, India
}

*Corresponding Author: Surender Kaur

Email: kaursurender@gmail.com

Received: $1^{\text {st }}$ May, 2018

Accepted: $1^{\text {st }}$ June, 2018

\begin{abstract}
Introduction and Objectives: The ESBLs producing organisms are reported worldwide in increasing numbers for which Clinical Laboratory Standard Institute (CLSI) recommends screening for ESBLs producing Escherichia coli and Klebsiella species by phenotypic methods. The study was undertaken to assess the burden of ESBL and comparison of Double Disc Synergy Test (DDST) and Phenotypic Confirmatory Test (PCT) with gold standard E-Test for ESBL detection in clinical isolates of tertiary care hospital, Bhopal.

Materials and Methods: 223 clinical isolates of E.coli and Klebsiellae species were included in the study and those showing zone size of $<22 \mathrm{~mm}$ for Ceftazidime were selected as potential ESBL producers. The ESBL were confirmed with gold standard E-test and later DDST \& PCT were performed over the suspected strains were compared with E-test. The data was maintained in MS Excel and appropriate tests of proportion and significance were applied.

Result: Out of 223 isolates 124 were potential ESBL producers with preponderance of E.coli 58.3\%. E-Test showed confirmed cases of ESBL 72/124(58\%) which was much higher than by DDST 44/124 (35.5\%) but close to PCT 70/124 (56.4\%) among suspected clinical isolates. Overall ESBL burden out of 223 isolates was $32.28 \%$ which is quite alarming. ESBL producing strain showed maximum sensitivity to Colistin, polymixin-B \& Imipenem nearing $100 \%$ whereas sensitivity ranging from 60 to $85 \%$ for beta lactamase inhibitor combinations. Non-ESBL producers were more sensitive to Amikacin, Fluoroquinolones, Nitrofurantoin, Cotrimoxazole with sensitivity ranging between 40 to $70 \%$.

Conclusion: With rise in ESBL producing strains with multi drug resistance, screening with Ceftazidime should be done to detect probable ESBL producer. PCT can be used with confidence with comparable results with that of MIC tests in resource poor microbiology laboratories which will save human, technical and monitory resources in terms of its confirmation by molecular genotypic methods.
\end{abstract}

Keywords: DDST-Double Disc Synergy Test, PCT-Phenotypic confirmatory test, E-Test-Epsilometer Test.

Key message: Screening with Ceftazidime and PCT for confirmation of ESBL should be routinely employed for resource poor microbiology laboratories for E.coli and Klebsiella species

\section{Introduction}

The most common organisms responsible for infections are multidrug resistant gram negative bacilli, particularly among members of the family Enterobacteriaceae \& non fermenting gram negative rods. Among the wide array of antibiotics, $\beta$-lactams are the most widely used agents. The most common cause of resistance to $\beta$-lactam antibiotics is the production of $\beta$-lactamases. The first plasmid-mediated $\beta$-lactamase in gram-negatives, TEM-1, was described in the early 1960s, originally found in a single strain of $E$. coli isolated from a blood culture from a patient named Temoniera in Greece, hence the designation TEM. Latter SHV-2, was found in a single strain of Klebsiella ozaenae isolated in Germany. Because of their increased spectrum of activity, especially against the oxyimino-cephalosporins, these enzymes were called extended-spectrum $\beta$-lactamases (ESBLs). ${ }^{1}$

Extended-spectrum $\beta$-lactamases (ESBLs), which hydrolyse extended-spectrum cephalosporins and are inhibited by $\beta$-lactamase inhibitors such as clavulanic acid, are spreading among Enterobacteriaceae. ${ }^{2}$ Plasmids coding for ESBLs may also carry additional $\beta$-lactamase genes as well as genes conferring resistance to other antimicrobial classes. They are usually associated with resistance to multiple unrelated antibiotics such as aminoglycosides, chloramphenicol, trimethoprim-sulfamethoxazole, tetracycline, and fluoroquinolones, leaving few therapeutic choices. ${ }^{3}$ Phenotypic detection of ESBLs among Enterobacteriaceae species is important for epidemiological purposes as well as for limiting the spread of resistance mechanisms. ESBL producing Enterobacteriaceae are now found in ambulatory patients without recognized risk factors for multidrugresistant organisms. ESBLs have emerged within the community, particularly among E.coli and $K$. pneumoniae. Various authors have reported the prevalence of ESBLs to be in the range of $6-88 \%$ in various hospitals, especially among Klebsiella pneumoniae and Escherichia coli. ${ }^{4}$ So the study was carried in E.coli and Klebsiella to look for the burden 
of ESBL in clinical isolates in central India with their antibiogram to aid the treating physicians in empirical therapy.

\section{Materials and Methods}

This cross sectional prospective analytical study was carried out in Department of Microbiology of Peoples College of Medical Sciences between November 2012 to April, 2014 over a period of one and half years. Clearance from research advisory committee and waiver of consent from Institutional Ethics committee for using the clinical samples received in department of Microbiology was obtained before processing of the samples. All the clinical samples were cultured and isolates identified according to standard microbiological techniques. ${ }^{5}$ A total of 223 Nonrepetitive isolates of E.coli and Klebsiella species from various clinical samples of urine, blood, pus, wound swab, sputum, intravenous catheter etc. from outpatient and inpatient units of all age groups and both sexes were included in the study.

The Antibiotic Sensitivity pattern was assessed using Kirby Bauer Disk Diffusion method for the drugs as per CLSI guidelines 2013. All the isolates having zone size of $\leq 22 \mathrm{~mm}$ for Ceftazidime were selected as potential ESBL producers being resistant to $3^{\text {rd }}$ generation cephalosporin for further processing confirming the ESBL producers by various phenotypic methods as per CLSI 2013 guidelines. ${ }^{6}$

\section{All potential ESBL producers were subjected to} three methods for detection of ESBL -

1. Double Disc Synergy Test (DDST)

2. National Committee for Clinical Laboratory Standards (NCCLS) Phenotypic confirmatory test (PCT)

3. MIC by Epsilometer Test (E-Test)

The test strain to be tested was inoculated in peptone water and incubated for 2 to $3 \mathrm{hrs}$. to be in log phase, The inoculum was adjusted to $0.5 \mathrm{McF}$ arland Turbidity standard using the Densimat ${ }^{\circledR}$ from BIOMERIEUX. The test strain was inoculated on 3 Muller Hinton Agar (MHA) Plates and disk placement done as follows and the plates were incubated overnight at $37^{\circ} \mathrm{C} \& \mathrm{read}$. The strain of Klebsiella pneumoniae ATCC 700603 was used as positive control whereas Escherichia coli ATCC 25922 was used as negative control while performing the tests.

1. For Double Disc Synergy Test (DDST) Amoxyclav disc $(30 \mu \mathrm{g}-20 \mu \mathrm{g}$ of amoxicillin \& $10 \mu \mathrm{g}$ clavulanic acid), Ceftazidime $(30 \mu \mathrm{g})$ and Cefotaxime $(30 \mu \mathrm{g})$ were used. With the help of a sterile forceps, Amoxyclav disc was placed in center of Muller Hinton agar plate. Ceftazidime and Cefotaxime discs were placed at $16 \mathrm{~mm}$ from Amoxyclav disc on either side. Accentuation of zone of cephalosporin disks towards clavulanic acid indicates ESBL production. ${ }^{7}$
2. For Phenotypic Confirmatory Test Ceftazidime (30 $\mu \mathrm{g})$ and Combination of Ceftazidime and Clavulanic acid $(30 / 10 \mu \mathrm{g})$ were placed opposite to each other in inoculated MHA. The measured zone of inhibition around Ceftazidime-clavulanic acid by more than $5 \mathrm{~mm}$ than that of Ceftazidime alone, confirms the isolate to be ESBL producer. ${ }^{7}$

3. For MIC by Epsilometric Test (E-test), E-test strips (TZ/TZL) obtained from BIOMERIEUX was placed on the third plate. It carries two gradients Ceftazidime $(0.5-32 \mu \mathrm{g} / \mathrm{ml}) \quad$ on one end \& Ceftazidime-clavulanic acid $(0.064-41 \mu \mathrm{g} / \mathrm{ml})$ in a different concentration gradient on other end. It had fixed concentration gradient of clavulanic acid (4 $\mu \mathrm{g} / \mathrm{ml})$. Manufacturer's instructions were followed for performing and interpreting the result. Presence of ESBL was confirmed by- ${ }^{8}$

i. Appearance of phantom zone.

ii. Or by deformation of Ceftazidime (TZ) eclipse.

iii. Or when MIC is reduced by $>3 \log _{2}$ dilutions.

iv. Or ratio of TZ/TZL $>8$ in presence of clavulanic acid as per manufacturer guide lines.

The E-test results were taken as confirmatory for detection of ESBL strain. The DDST and PCT were then compared with the gold standard test for their overall utility as phenotypic tests in routine bacteriology practices. All the data was maintained in Microsoft office Excel and was analyzed using test of proportion and test of significance.

\section{Result}

A total of 223 isolates of Escherichia coli and Klebsiella species from different clinical samples were enrolled for the study. Maximum isolates were received from young adults between 21-30 year $(24.7 \%)$ and patients aged $>61$ year (22.9\%), with insignificant gender distribution. Maximum isolates were from admitted patient's i.e. $80.7 \%$.

A total of 124 of $223(55.60 \%)$ isolates were suspected to be possessing the ESBL based on the reduced susceptibility shown to Ceftazidime with $\leq 22$ $\mathrm{mm}$ zone of inhibition. Speciation of the same along with the total number of confirmed cases are as depicted in Table 1. A total of 72/223 (32.28\%) strains were confirmed to be ESBL producer by Gold standard test i.e. MIC by E-test. 
Table 1: Speciation of isolated Gram negative bacilli and suspected and confirmed ESBLs by gold standard

\begin{tabular}{|l|c|c|c|}
\hline \multicolumn{1}{|c|}{ Clinical Isolates } & $\begin{array}{c}\text { Speciation of Isolated GNB } \\
\text { Percent (\%) }\end{array}$ & $\begin{array}{c}\text { Suspected ESBL } \\
\text { producers }\end{array}$ & $\begin{array}{c}\text { Confirmed ESBL by } \\
\text { Gold Standard }\end{array}$ \\
\hline Escherichia coli & $144(64.6 \%)$ & $84(58.3 \%)$ & 50 \\
\hline Klebsiella pneumoniae & $74(33.2 \%)$ & $38(51.3 \%)$ & 22 \\
\hline Klebsiella oxytoca & $05(02.2 \%)$ & $02(40 \%)$ & 00 \\
\hline Total & $(223) 100.0 \%$ & $124(55.6 \%)$ & 72 \\
\hline
\end{tabular}

The clinical sample wise distribution of the enrolled, suspected and confirmed cases of the E.coli and Klebsiella species is depicted in Table 2. Urine was the most predominant sample received which yielded 95/223 (42.60\%) followed by 51/223 (22.86\%) and $30 / 223(13.45 \%)$ for pus and sputum respectively.

Table 2: Clinical sample wise distribution of the enrolled, suspected and confirmed cases of the E.coli and Klebsiella species

\begin{tabular}{|l|c|c|c|c|c|c|c|c|}
\hline \multicolumn{1}{|c|}{ Sample } & Urine & Pus & Sputum & Tips & HVS & Blood & $\begin{array}{c}\text { Body } \\
\text { fluids }\end{array}$ & Total \\
\hline E.coli detected & 75 & 19 & 18 & 15 & 12 & 03 & 02 & 144 \\
\hline Suspected ESBL & 43 & 10 & 09 & 07 & 10 & 03 & 02 & 84 \\
\hline ESBL by Gold standard test & 29 & 07 & 06 & 01 & 05 & 00 & 02 & 50 \\
\hline Klebsiella species detected & 20 & 32 & 12 & 09 & 05 & 00 & 01 & 79 \\
\hline Suspected ESBL & 08 & 17 & 07 & 04 & 03 & 00 & 01 & 40 \\
\hline ESBL by Gold standard test & 03 & 09 & 06 & 02 & 02 & 00 & 00 & 22 \\
\hline
\end{tabular}

The maximum samples were received from intensive care unit 51(22.86\%) followed closely by Surgical and Outpatients department with $42(18.83 \%)$ and $43(19.28 \%)$. The department wise distribution of the samples received and enrolled, their species wise isolation and response to various phenotypic tests along with the gold standard is shown in table 3 .
The maximum isolation of confirmed cases of ESBL were from the Surgery department with $18 / 72(25 \%)$ with $15(20.83 \%) \& 12(16.66 \%)$ cases from OPD and pulmonary medicine department. Whereas from Medicine department there were 10/72 (13.88\%) confirmed cases.

Table 3: Department wise distribution of samples yielding E.coli and Klebsiella species along with suspected ESBL producing strains tested and confirmed by various phenotypic methods.

\begin{tabular}{|l|c|c|c|c|c|c|c|c|c|}
\hline Department & $\begin{array}{c}\text { Sample } \\
\text { No(\%) }\end{array}$ & \multicolumn{2}{|c|}{ E.coli } & \multicolumn{2}{c|}{ Klebsiella species } & Total & E Test & PCT & DDST \\
\hline & & Isolated & Suspected & Isolated & Suspected & & & & \\
\hline I.C.Us. & $51(22.86 \%)$ & 39 & 15 & 12 & 03 & 51 & 07 & 07 & 04 \\
\hline Burn & $05(02.2 \%)$ & 01 & 01 & 04 & 03 & 05 & 02 & 02 & 03 \\
\hline Medicine & $19(08.5 \%)$ & 13 & 09 & 06 & 04 & 19 & 10 & 10 & 06 \\
\hline Surgery & $42(18.83 \%)$ & 21 & 18 & 21 & 11 & 42 & 18 & 17 & 10 \\
\hline Gynecology & $26(11.7 \%)$ & 17 & 09 & 09 & 03 & 26 & 04 & 04 & 05 \\
\hline $\begin{array}{l}\text { Pulmonary } \\
\text { medicine }\end{array}$ & $27(12.1 \%)$ & 14 & 10 & 13 & 08 & 27 & 12 & 12 & 07 \\
\hline Pediatric & $10(04.4 \%)$ & 06 & 04 & 04 & 04 & 10 & 04 & 04 & 02 \\
\hline OPD & $43(19.28 \%)$ & 33 & 18 & 10 & 04 & 43 & 15 & 14 & 07 \\
\hline Total & 223 & 144 & 84 & 79 & 40 & 223 & 72 & 70 & 44 \\
\hline
\end{tabular}

The DDST and PCT when compared with the gold standard test i.e. E-test and though both the tests showed $\mathrm{p}$ value of less than 0.001 and were highly significant for detection of the ESBL, the PCT with sensitivity of more than $97 \%$ and specificity of 100 percent is found to be comparable to gold standard with Positive predictive value of $100 \%$ and negative predictive value of $96.2 \%$ as depicted in Table 4 . 
Table 4: Comparative evaluation of DDST \& PCT against E-Test with Sensitivity, Specificity, NPV\& PPV

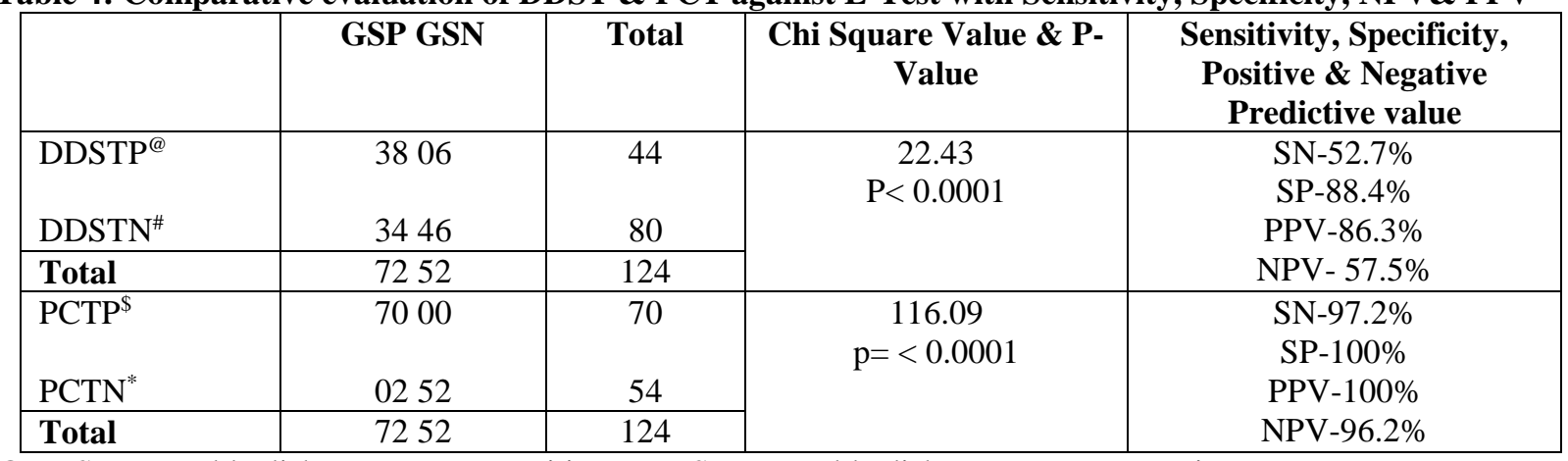

@DDSTP- Double disk synergy test positive \# DDSTN- Double disk synergy test negative

$\$$ PCTP- Phenotypic confirmatory test positive *PCTN- Phenotypic confirmatory test negative,

SN-Sensitivity, SP-Specificity, PPV- Positive predictive value \& NPV- Negative predictive value

The antibiogram of the confirmed ESBL producing strain was compared with the Non- ESBL producing strain (Graph 1) ESBL producing strain showed maximum sensitivity to Colistin, polymixin-B \& Imipenem nearing $100 \%$ whereas sensitivity ranging from 60 to $85 \%$ for beta lactamase inhibitor combinations. These strains were not much sensitive to
Amikacin, Fluoroquinolones, Nitrofurantoin, Cotrimoxazole with a sensitivity below $30 \%$.

The Non-ESBL producer strains when compared to ESBL producing strain showed comparable sensitivity to Colistin, polymixin-B \&Imipenem as well as beta lactamase inhibitor combinations. They were more sensitive to Amikacin, Fluoroquinolones, Nitrofurantoin, Cotrimoxazole with sensitivity ranging between 40 to $70 \%$. (Graph 1)

\section{Graph 1}

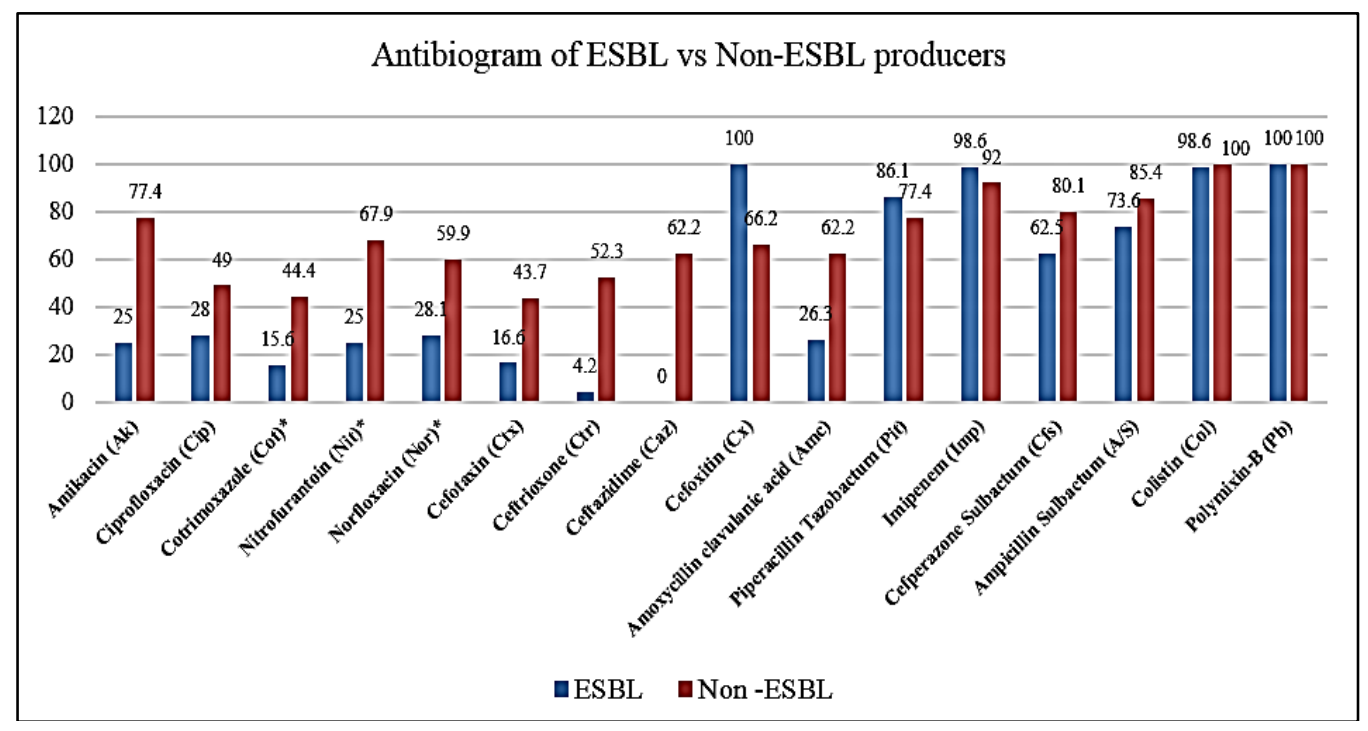




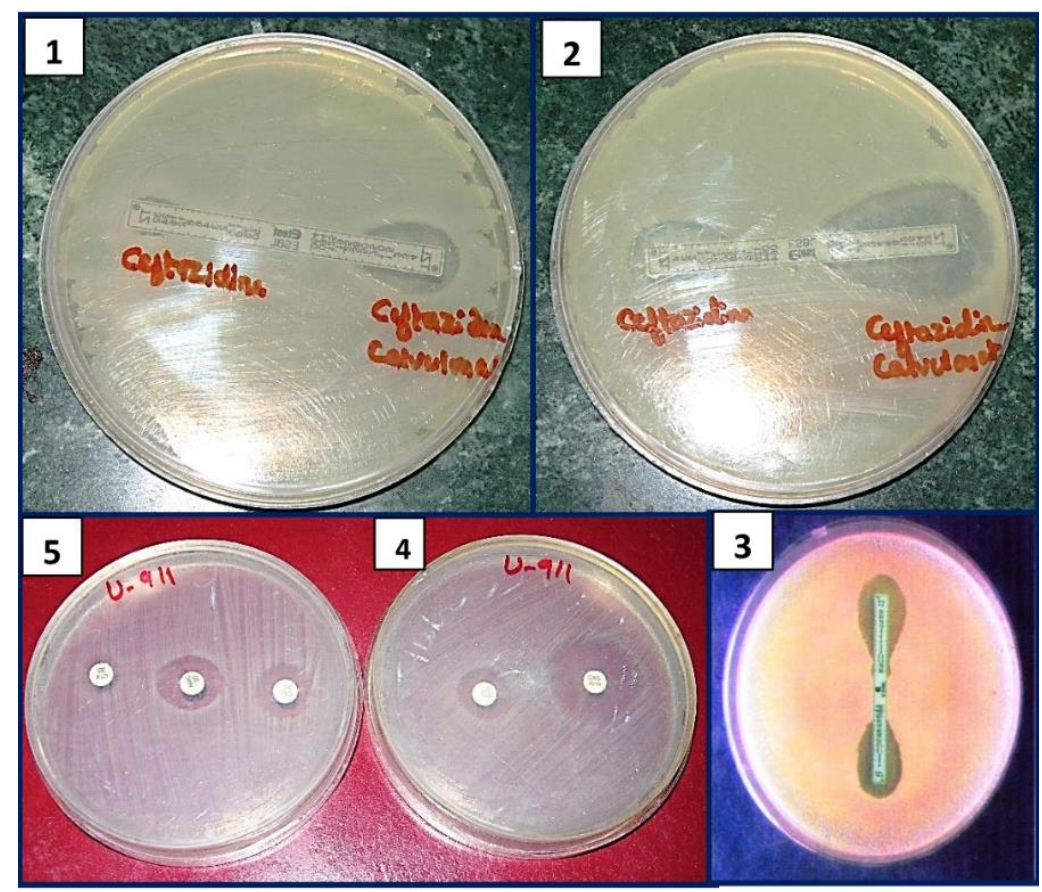

Fig. 1-3: $E$ test and 4-DDST test and 5-PCT test positive for ESBL

\section{Discussion}

In recent years there has been an increase incidence and prevalence of ESBLs; enzymes that hydrolyze and cause resistance to oxyimino-cephalosporin's and aztreonam. ${ }^{2}$ These enzymes are most commonly produced by Klebsiella pneumoniae, Klebsiella oxytoca and E.coli. ${ }^{9}$ So ESBL detection was done in isolates in Klebsiella species and E.coli in this study. CLSI standard disc diffusion test was used as a screening test to detect probable ESBL producer, followed by confirmatory Gold standard test i.e. E-test and comparison of Double disk synergy test (DDST) \& Phenotypic confirmatory test (PCT) with E-test.

Detection of Potential ESBLs: In our study, we found $55.6 \%$ potential ESBLs out of total 223 Gram negative isolates (E.coli and Klebsiella species) (Table 1) which was in accordance with Veena Krishnamurthy et al ${ }^{10}$ who found $59.7 \%$ potential ESBLs, with predominance of E.coli. As per CLSI guidelines we used Ceftazidime for screening ESBLs, Katsanis GP et $\mathrm{al}^{11}$ also says Ceftazidime is the best single test antibiotic for detecting ESBL production.

Confirmation test for ESBL -by E-test: In this study E-test was used as a gold standard method for confirmation of ESBL producers by MIC method. Etest was able to detect $72 / 223$ (32.28\%) enrolled strains whereas $72 / 124(58.06 \%)$ of the suspected ESBL strain. 50/72(69.44\%) were E.coli whereas 22/72(30.55\%) were Klebsiella pneumoniae. No Klebsiella oxytocaisolate was detected as ESBL producer. (Table 2) Maurine et $\mathrm{al}^{12}$ and Anandkumar et $\mathrm{al}^{13}$ have also used E-Test as the reference for detection of ESBL with up to $94 \%$ accuracy.
The overall prevalence of ESBL producers was found to vary greatly in different geographical areas and in different institute in India, based on various risk factors, local reasons and rationale use of $\beta$-Lactam antibiotics. It is high in referral centers where antibiotic use is profuse. In a study by wattle C. et al. ${ }^{14}$ in Delhi, the prevalence was as high as $91.7 \%$ which was probably due to high use of cephalosporin's, high rate of patient transfer from peripheral centers and associated patient risk factors like chronic ill health etc.

In the present study, out of 223 isolates $32.28 \%$ were ESBL producers which was in accordance with Ranjan $S^{15}$ (2012), $34.8 \%$ but less than that of Babypadmini et al (2004), ${ }^{16} \mathrm{C}$ Rodrigues et al (2004) ${ }^{17}$ and G Dalella(2011) ${ }^{18}$ with $40.3 \%, 53 \%$ and $61.6 \%$ respectively.

Male to female ratio observed was 1:1.4 which was higher as compared to Babypadmini et al ${ }^{16}$ (2004) 1:1.3 and Nema Shashwati ${ }^{19}$ (2014)1:1.2 but common in all studies were female predominance.

In the present study, maximum number of ESBL producers were inpatients $(79.1 \%)$ and minimum were outpatients $20.8 \%$, which was in accordance to Avinash Laghawe et $\mathrm{al}^{20}(2012)$ where they were $85.94 \%$ and $14.06 \%$ respectively.

In our study, ESBL producers were more often isolated from urine (44.4\%) followed by pus $(22.2 \%)$ and sputum (16.7\%). Yazdi et $\mathrm{al}^{21}$ by phenotypic method confirmed $44.3 \%$ ESBL among uropathogens.

There is a rising incidence of UTI with ESBL producing bacteria. Hence, routine ESBL testing for uropathogens along with conventional antibiogram would be useful for all cases of UTI. (Table 2) 


\section{Comparative Evaluation}

Other Phenotypic methods like DDST and PCT were then compared to the Gold standard method for detection of the ESBL. (Table 4) DDST could detect 44/124 (35.48\%) suspected strain to be ESBL as against $72(58.06 \%)$ by E-test. Our study showed that 28 stains could not be detected by DDST. Similar results were found by Renuka Rampure et $\mathrm{al}^{22} \&$ Martin et $\mathrm{al}^{23}$ whereas Jasmine et $\mathrm{al}^{24}$ found equivocal result between E-test and DDST. Cormican et $\mathrm{al}^{25}$ reported DDST 79\% sensitive as compared to E-test, having itself $100 \%$ sensitivity. Although E-test is expensive, yet many investigators reported it to have high sensitivity and to be convenient. Though comparable with $\mathrm{p}$ value of < 0.001 , the sensitivity of $52.7 \%$ doesn't make it a good test to rely upon. ${ }^{18}$ DDST lacks sensitivity because of optimal disc spacing and incorrect storage of clavulanate containing disc. ${ }^{26}$

On comparing E-test with PCDT, only 2 more isolates were detected by E-test and performance of PCT was found to be in agreement with the Gold standard test. (Table 4) Present study showed at par result similar to Gaurav Dalela ${ }^{18}$ and Derek et $\mathrm{al}^{27}$ and Anbumani Narayanaswamy ${ }^{28}$ found $100 \%$ agreement of the two methods- phenotypic confirmatory test and Etest strips in detection of ESBL producers. Enas Sh. Khater et $\mathrm{al}^{29}$ (2014) also says E-test to be more sensitive.

The discovery and development of antibiotics was undoubtedly one of the greatest advances of modern medicine. Unfortunately, the emergence of antibiotic resistance bacteria is threatening the effectiveness of many antimicrobial agents which has not only increased hospital stay but also economic burden of patients. The production of beta-lactamase may be of chromosomal or plasmid origin. Plasmid mediated production is often acquired by transfer of genetic information from one organism to another. Such transferable plasmid also codes for resistant determinants to other antimicrobial agents. Hence multidrug resistance is expected to be more common in ESBL producing organisms. ${ }^{30}$

In our study, resistance among ESBL producer for cephalsporins, Cefotaxime 83.3\%, Ceftrioxone 95.8\% and Ceftazidime $100 \%$ where as Ciprofloxacillin and Amikacin showed $81.9 \%$ and $44.4 \%$ respectively. Resistance among uropathogen ESBL producers were for Cotrimoxazole $84.3 \%$, Nitrofurantoin $75 \%$ and Norfloxacin $71.8 \%$. The non ESBL producers had different mechanisms for their resistance pattern. (Graph 1) For other antibiotics, the resistance pattern was found to be more in the ESBL producers as compared to their non-ESBL producing counterparts. Hence forth, ESBL producing isolates were resistant to more antimicrobial agents than non-ESBL producing isolates.

In our study Susceptibility for Imipenemwas 98.6\% and for piperacillin/tazobactum 76.3\%. Henceforth, Imipenem is the most active drug for the treatment of infections caused by ESBL producers, followed by piperacillin/tazobactum and amikacin. We need to keep in mind that carbapenem must be kept in reserve for life-threatening infections where other susceptible antibiotics can be used. Carbapenems are the mainstay of therapy but, they are expensive and require prolonged intravenous administration and there is growing concern of carbapenem resistance in clinical isolates.

\section{Limitations of Study}

The important limitations of all the phenotypic tests based on synergy is their inability to detect OXA and Amp C enzymes which are of growing concern. Hyper production of TEM and/or SHV $\beta$-lactamases if accompanied with ESBL can cause false negative result by phenotypic test.

\section{Conclusion}

Keeping in view the growing incidences of the Multi Drug Resistant Organisms, the Gram negative clinical isolates should be looked for the presence of ESBL production and associated other resistance mechanisms. Resistance to Ceftazidime should be taken as a probable production of ESBL which can be confirmed by PCT effectively in resource poor microbiology laboratories or by MIC by E-test which is a bit costlier but definitely will save human, technical and monitory resources in terms of its confirmation by molecular genotypic methods.

\section{Acknowledgement}

I would like to acknowledge precise contribution of Dr. Naveenchandra M Kaore who provided insight and expertise in guiding me in meticulous analysis of work and scientific writing.

\section{References}

1. Calil R, Marba ST, Tresoldi AT. Reduction in colonization and nosocomial infection by multi resistant bacteria in neonatal unit after institution of educational measures and restriction in the use of cephalosporins. Infect Control Hospital Epidemiol. 2001;29:133-8.

2. Bradford PA. Extended-spectrum $\beta$-lactamases in the $21 \mathrm{st}$ century: characterization, epidemiology, and detection of this important resistance threat. Clin Microbiol Rev. 2001;14:933-951.

3. Jacoby GA, Medeiros AA. More extended-spectrum $\beta$ lactamases. Antimicrob Agents Chemother. 1991;35:1697-1704.

4. Rodriguez-Bano J, Acala JC, Cisneros JM, Grill F, Oliver A, Horcajada JP, Tórtola T, Mirelis B, Navarro G, Cuenca M, Esteve M, Peña C, Llanos AC, Cantón R, Pascual A. Community infections caused by extendedspectrum beta-lactamase-producing Escherichia coli. Arch Intern Med. 2008;168:1897-1902.

5. Collee JG, Miles RS, Watt B. Tests for identification of bacteria. In, Collee JG, Marmion BP, Fraser AG, Simmons A (eds).Mackie and McCartney Practical Medical Microbiology. 14th Edition. Delhi, Churchill Livingstone,2007;131-49. 
6. Clinical and laboratory standards institute. Performance standards for antimicrobial susceptibility testing twenty third informational supplemented. CLSI document M100S23.Wayne, PA:CLSI;2013.

7. Shukla I, Tiwari R, Agrawal M. Prevalence of extended spectrum $\beta$ lactamase producing klebsiella pneumoniae in a tertiary care hospital. IJMM. 2004;22(2):87-91.

8. AB BioMerieux, Solna, Sweden.

9. Konean EW, Allen SD, Janda WM, Schreckenberger Pc, Winn WC. Antimicrobial susceptibility testing In Color atlas and textbook of diagnostic microbiology, $5^{\text {th }} \mathrm{Ed}$. Lippincott Williams and wikins Company 1997; pp 786, 798-801, 808, 831-832.

10. Veena Krishnamurthy, Vijay Kumar G.S, Sudeepa Kumar M, Prashanth, Prakash R, Nagraj E R. Phenotypic and Genotypic methods for detection of extended spectrum $\beta$-lactamase in Escherichia coli and Klebsiella pneumoniae isolated from ventilator associated pneumoniae. Journal of Clinical and Diagnostic Research. 2013;7(9):1975-1978.

11. Katsanis GP, Spargo J Ferraro, MJ Sutton L, Jacoby GA. Detection of Klebsiella pneumoniae and Escherchiae coli strains producing extended spectrum $\beta$-lactamases. $J$ Clin Microbiol. 199;32(3):691-696.

12. Maurine A. Leverstein-van Hall, Ad C. Fluit, Armand Paauw, Adrienne T. A. Box, Sylvain Brisse, and Jan Verhoef. Evaluation of the Etest ESBL and the BD Phoenix, VITEK 1, and VITEK 2 Automated Instruments for Detection of Extended-Spectrum Beta-Lactamases in Multiresistant Escherichia coli and Klebsiella spp. Journal of Clinical Microbiology. 2002:3703-3711.

13. Anandkumar Harwalkar, Jagadeesh Sataraddi, Soham Gupta, Raksha Yoganand, Achut Raoa, Hiresave Srinivasa. The detection of ESBL-producing Escherichia coli in patients with symptomatic urinary tract infections using different diffusion methods in a rural setting. Journal of Infection and Public Health. 2013;6:108-114.

14. Wattal C, Chugh TD, Sharma A, Overoi JK, Datta S. Microbiology Newsletter Sir Ganga Ram Hospital, 2005;10(1):1-8.

15. Rajan S, Prabavathy J. Antibiotic Sensitivity and Phenotypic Detection of ESBL producing E.Coli Strains Causing Urinary Tract Infection In a Community Hospital, Chennai, Tamil Nadu, India. Webmed Central> Research articles 2012;11:1-15.

16. Baby Padmini S, Appala Raju B, Mani KR. Detection of Enterobacteriaceae producing CTX-M Extended spectrum beta-lactamases from Tertiary care Hospital in south India. IJMM. 2008;26(2):163-6.

17. C Rodrigues, $\mathrm{P}$ Joshi, SH Jani, M Alphonse, $\mathrm{R}$ Radhakrishnan, A Mehta. Detection of -Lactamases in nosocomial gram negative clinical isolates. IJMM. 2004;22(4):247-250.

18. Gaurav Dalela. Prevalence of Extended Spectrum Beta Lactamase (ESBL) Producers among Gram Negative Bacilli from Various Clinical Isolates in a Tertiary Care Hospital at Jhalawar, Rajasthan India. Journal of clinical \& Diagnostic Research. 2012;6(2):182-87.

19. Nema Shashwati, Tripathi Kiran, A. G. Dhanvijay Study of extended spectrum $\beta$-lactamase producing Enterobacteriaceae and antibiotic coresistance in a tertiary care teaching hospital. Journal of Natural Science, Biology and Medicine. 2014;5(1):30-35.
20. Avinash Laghawe, Neelam Jaitly, Vilas Thombare. The Simultaneous Detection of the ESBL and the AmpC bLactamases in Gram Negative Bacilli. Journal of Clinical and Diagnostic Research. 2012;6(4):660-663.

21. Yazdi M, Nazemi A, Mirinargasi M, Jafarpour M, Sharifi SH. Genotypic versus Phenotypic methods to detect Extended-Spectrum Beta-Lactamases (ESBLs) in Uropathogenic Escherichia coli Scholars Research Library Annals of Biological Research. 2012;3(5):24542458.

22. Renuka Rampure, Ravindranath Gangane, Ajay Kumar Oli1, Kelmani Chandrakanth R. Prevalence of MDRESBL producing Klebsiella pneumoniae isolated from clinical Samples. J Microbiol Biotech Res. 2013;3(1):3239.

23. Martin G, Cormican, Steven A, Marshall, and Ronald N, Jones. Detection of ESBL producing strains by the E-test ESBL screen. $J$ of Clin. Micro. 1996:1880-1884.

24. Jasmine Subashini and Krishnan Kannabiran. Screening and Identification of Extended Spectrum $\beta$-lactamase (ESBL) Pathogens in Urine Sample of UTI Patient's. Trop Med Surg. 2013:1:3.

25. Cormican MG, Marshall SA, Jones RN. Detection of Extended-Spectrum $\square$-Lactamase (ESBL) producing strains by the E test ESBL screen. J ClinMicrobiol. 1996;34:1880-1884.

26. P.L. Ho, K.H. Chow, K.Y. Yuen, W.S. Ng, P.Y. Chau Comparison of a novel, inhibitor - potentiated disc diffusion test with other methods for the detection of extended-spectrum $\beta$-lactamases in Escherichia coli and Klebsiella pneumonia. Journal of Antimicrobial Chemotherapy. 1998(42):49-54.

27. Derek FJ, Brown, Jenny Andrews, Anna King and Alasdair P, MacGowan. Detection of Extended spectrum $\beta$-lactamase with E-test and double disc potentiation methods. Journal of Antimicrobial Chemotherapy. 2000;46(2):327-328.

28. Anbumani Narayanaswamy and M. Mallika Prevalence and Susceptibility of extended spectrum beta-lactamases inurinary isolates of Escherichia coli in a Tertiary Care Hospital, Chennai-South India. Internet Journal of Medical Update. 2011;6(1):39-43.

29. Enas Sh. Khater and Hammamouda W. Sherif. Rapid detection of extended spectrum $\beta$ lactamase (ESBL) producing strain of Escherichia coli in urinary tract infections patients in Behna university hospital, Egypt. British microbiology Research Journal. 2014;4(4):443445.

30. Shields J, Maxwell AP. Acute pyelonephritis can have serious complications. Practitioner 2010;254:19, 21, 23$4,2$.

How to cite this article: Kaur S, Awari A. Burden of ESBL with antibiogram in clinical isolates of E.coli and Klebsiella species from tertiary care hospital in central India. Indian J Microbiol Res. 2018;5(4):497503. 